\title{
Generalised Hypercementosis in a Young Female Seeking Extraction: Revision and Update of Surgical Technique
}

\author{
Shadia Elsayed1, Albraa Alolayan1, Lamees Farghal2 and Yousra Ayed 3 \\ Department of Oral and Maxillofacial Surgeryl / Oral Surgery2/ Oral Basic Science3, Taibah University Dental College and Hospital, \\ Al-Madinah Al-Munawwarah 42354, Saudi Arabia
}

\begin{abstract}
Hypercementosis is an excessive cementum deposition at the tooth root apex which occurs pathologically and represents as a body reaction to various stimulating factors including infection or endodontic treatment in most cases. Although hypercementosis mostly complicates teeth extraction, it is rare in daily dental practice. In the present case report, a generalised maxillary and mandibular hypercementosis in a young female patient is represented with a detailed description of its histologic, radiographic, and clinical characteristics followed by a review of surgical technique. It is clinically relevant to correlate hypercementosis of teeth, the feasibility of the best surgical method, in order to achieve a safe uncomplicated extraction.
\end{abstract}

Key Words: Generalised hypercementosis, Extraction, Teeth.

How to cite this article: Elsayed S, Alolayan A, Farghal L, Ayed Y. Generalised hypercementosis in a young female seeking extraction: revision and update of surgical technique. J Coll Physicians Surg Pak 2019; 29(11):1111-3.

\section{INTRODUCTION}

Although hypercementosis is a rare incidental phenomenon that mostly complicates teeth extraction. It is characterised by excessive deposition of cementum at the tooth apex, mostly idiopathic in nature. It is asymptomatic and usually discovered during a radiographic examination or during difficult extraction visit. Hypercementosis does not cause any changes in the periodontal ligament, biologic width or alveolar bone around the root's surfaces. ${ }^{1}$

Presentation of generalised hypercementosis is not very common in the literature. Hereditary or systemic disturbances are considered to be etiological factors. The hypercementosis diagnosis is based on the characteristic radiographic radiopacity present at the tooth root. ${ }^{2}$

Once hypercementosis is diagnosed, considerable precautionary measures and care should be taken for extraction to avoid complications owing to this condition.

Additionally, it creates anxiety among the surgeons, although it is not a tumor that invades the bone. It is depiction of extra cementum with each new layer being deposited over the previous layer around the root apex without invading the neighbouring bone, which usually requires no treatment. 3

Correspondence to: Dr. Yousra Ayed, Department of Oral Basic Science, Taibah University Dental College and Hospital, Al-Madinah Al-Munawwarah 42354, Saudi Arabia

E-mail: yosraayed82@gmail.com

Received: December 13, 2018; Revised: March 2, 2019;

Accepted: March 28, 2019
Sampieri et al. reported that in cases of hypercementosis, there is a high resistance during tooth extraction. However, in reviewing most oral and maxillofacial surgery recommendations and references, there is inadequate information about the best method of extraction of this ball attached to the root safely and the only advice given is to be cautious during extraction. ${ }^{4}$

In Saudi population in Sakaka, Aljouf, the frequency of hypercementosis is low (4.8\%) as reported by Patil et al., in 2018: whereas, in Almadinah Almunawwarah, hypercementosis may be a relatively common finding, and its influence on the treatment of teeth affected by this alteration should be realised by the dentists. ${ }^{5}$

Therefore, the purpose of the current report is to introduce this case of hypercementosis, review and update the recommended surgical techniques used for extraction, and to explore more avenues on the surgical treatment of hypercementosed teeth with the least complication.

\section{CASE REPORT}

A 38-year female was referred from the Restorative Department for extraction of an unrestorable tooth. The patient was referred to an oral and maxillofacial surgery clinic to complete the case rehabilitation.

The patient's medical history was clear. On preoperative clinical examination, there were multiple attrited teeth with mild gingivitis with no other abnormal features. Upper left third molar was indicated for extraction due to chronic periodontitis and over-eruption as no opposing tooth was present.

From the orthopantomogram radiograph, a generalised hypercementosis was noticed in almost all the maxillary 


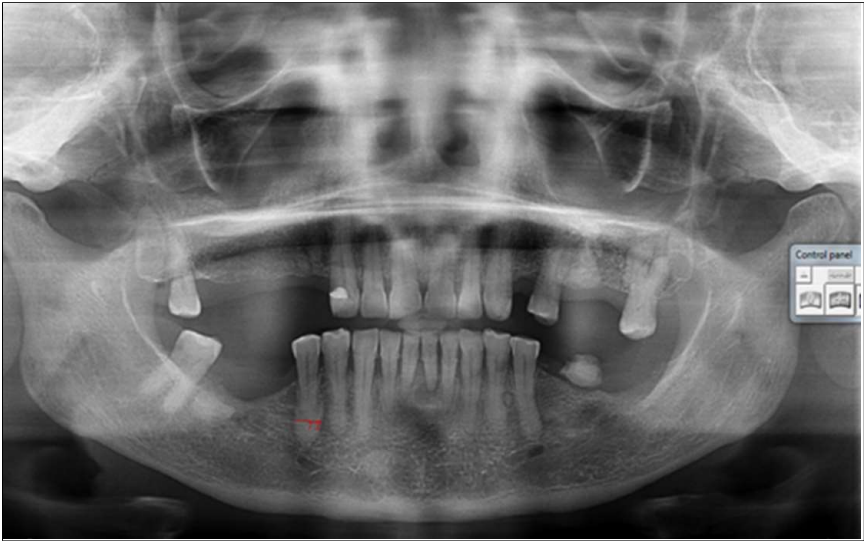

Figure 1: Orthopantomogram of the patient showing multiple teeth affected with apical hypercementosis and also separated cementum-like lesions at other edentulous areas.

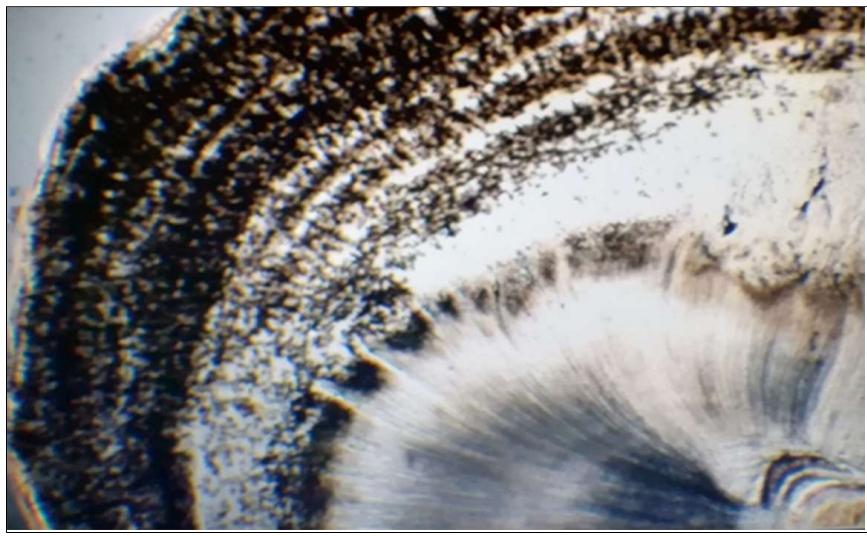

Figure 2: Ground section demonstrating thick layer of cementum. The primary viable cementum rich in cementocytes lacunae and canaliculi; whereas, the last layer is the secondary cementum that appears darker (under $100 \times$ magnification)

and mandibular teeth (number 21, 25, 28, 35, 34, 33, 43, 44,45 , and 48). The root apices were grossly thickened and blunted due to a fairly symmetrical deposition of a radiopaque material on their apical surfaces (Figure 1). Attrition was noticed in the lower anterior and premolar teeth. The following teeth were missing $(15,16,17,24,27$, $36,37,38,46$, and 47 ). There were retained radiopaque cementum-like lesions attached to the distal surface of upper left first molar and isolated cementum-like lesions in the right and left mandibular molar region and reduction of the height of the alveolar process in the relevant area of the missing teeth with a generalised interdental bone loss. Hypercementosis measurements were conducted on the Care Stream R4 Kodak software (Carestream Dental, Herts, UK). The width ranged from 4 to $7 \mathrm{~mm}$ and digital radiodensity ranged from 119 to 155 pixels.

We chose to start by normal extraction movements which reached maximum socket expansion and the tooth was loose but did not pull outside the socket. Mucoperiosteal flap was not used. Normal movement of extraction was started, then the resistance was decreased by space guttering parallel to the roots long axis in depth of the buccal bone side at the expense of root structure and this was sufficient to aid in luxation plus sectioning of the roots vertically. The interseptal bone removal was needed down to the hyper-cementosed root apex level in order to allow retrieval of the root. This led to an excessive widening of the socket and removal of the root easily using wide tip straight apexo elevator. Uneventful healing of the extraction socket occurred in usual time without any complication. Pain and edema were as usually accompanied by normal exodontia patient. Macroscopically, following extraction, a yellowish calcified hard substance with an irregular surface could be observed in the apical area of the extracted tooth.

To establish a definitive diagnosis, the tooth specimen was sent for histopathological examination. The extracted tooth parts were fixed in $4 \%$ buffered paraformaldehyde. The tooth was embedded in methyl methacrylate and cut horizontally into ground sections using a slow-speed diamond saw (Varicut VC-50; Leco, Munich, Germany). The root surfaces were observed histologically by microscopy (OLYMPUS BX71, Tokyo, Japan) and this confirmed the diagnosis of hypercementosis. A high number of cementocytes and cementocytes lacunae in the area with hypercementosis appeared in the tissue sections. Numerous canaliculi can be seen where a number of cytoplasmic extensions were irregularly distributed and communicated with other cementocytes (Figure 2).

\section{DISCUSSION}

Radiographic examination revealed generalised hypercementosis affecting both mandibular and maxillary teeth. As we approached this referred surgical extraction case, we did not find in literature, a complete detailed description of surgical extraction technique of hypercementosed tooth although it is mentioned obviously in all oral surgery guidelines and references, thus it needs special care for surgical exodontia. ${ }^{6}$

The problem in extracting hypercementosed tooth is the risk for higher complications such as fracture tuberosity or weakening of the mandible, causing a high risk of jaw fracture; so special precaution and informed consent from the patient before surgery is mandatory. In some reported cases, fusion of the hypercementosis tooth to the neighbouring teeth forming concrescence occurs which leads to difficult combined extraction. Suter et al. reported severe hypercementosis of four maxillary premolar and molar teeth, which led to concrescence of all the segments. ${ }^{7}$

Hypercementosis can be radiographically identified, but it is not possible to determine the exact amount of cementum present in the affected roots because dentin and cementum have the same radiodensity. Therefore, a microscopic examination determines more accurately the exact amount of cementum. ${ }^{8}$

Furthermore, generalised hypercementosis can either be of the dense type or the transparent one. The present case of generalised hypercementosis was asymptomatic 
and discovered incidentally. It was a transparent type, and the demarcation between the secondary and primary cementum and dentin was clearly visualised. A high number of cementocytes and cementocytes lacunae were seen in primary cementum (Figure 2). ${ }^{9}$

The etiology of generalised hypercementosis can be categorised as hereditary and systemic conditions such as Paget's disease, thyroid goiter, rheumatic fever, rheumatoid arthritis, and acromegaly. Even trauma or minor trauma has been reported to be associated with this condition. Reactional hypercementosis appears to be stimulated by endodontic or periodontal problems which cannot explain cases with generalised hypercementosis that affect all teeth in both upper and lower jaws.

However, on reviewing different oral and maxillofacial surgery guidelines, there is a lack of detailed studies guiding surgical extraction in teeth with hyper-cementosis. Fragiskos in 2007, described normal three-line or envelope flap and removal of the buccal bone in single or multirooted teeth and discussed how to remove the interseptal bone with widening of the sockets and then removal of the root. 10

In this case, we did all the above steps, except that we did not reflect a flap and we used conservative guttering of the buccal bone only using parallel fissure bur. Furthermore, complete removal of the buccal bone was not performed; however, there is a need in such cases for removal of the interseptal bone completely down to the level of the hypercementosed root apex.

It was noted in contrary to the Fragiskos's description that hypercementosed tooth roots were not liable to fracture at the root apex, as in forcible extraction of other normal premolars as we noticed that the hypercementosed tooth was very strong. ${ }^{10}$

Additionally, hypercementosis has an effect on teeth movement during orthodontic treatment. Extraoral extraction technique may be an option to extract a hypercementosed tooth. Others think that coronectomy is the best choice in the extraction of such tooth. One case report showed hypercementosis of second upper molar roots and an atypical fusion to the crown of an unerupted third molar. If such unions are not recognised before tooth extraction, complications such as maxillary tuberosity and fracture with extended oro-antral fistulas may occur. 9,10

The present report described a case of generalised hypercementosis referred to oral surgery clinic for extraction. The purpose of current report is to review and emphasise on how to remove hypercementosed teeth safely and professionally. Further studies are required to determine the prevalence and pattern of hypercementosis to increase the awareness of oral surgeons and determine the optional method for these difficult extraction situations.

\section{CONFLICT OF INTEREST:}

Authors declared no conflict of interest.

\section{AUTHORS' CONTRIBUTION:}

SE: Designed and edited the manuscript; reviewed and finally approved the manuscript.

AA, LF, YA: Data collection, drafting and manuscript writing; review and final approval of manuscript.

\section{REFERENCES}

1. Bürklein S, Jansen $S$, Schäfer $E$. Occurrence of hypercementosis in a German population. J Endod 2012; 38:1610-2.

2. Zhou J, Zhao Y, Xia C, Jiang L. Periodontitis with hypercementosis: Report of a case and discussion of possible aetiologic factors. Aust Dent J 2012; 57:511-4.

3. D'Incau E, Couture C, Crépeau N, Chenal F, Beauval C, Vanderstraete $\mathrm{V}$, et al. Determination and validation of criteria to define hypercementosis in two medieval samples from France (Sains-en-Gohelle, AD $7^{\text {th }}-17^{\text {th }}$ century; Jau-Dignac-etLoirac, AD 7th-8th century). Arch Oral Biol 2015; 60:293-303.

4. Da Silva Sampieri MB, Viana FLP, Cardoso CL, Vasconcelos MF, Vasconcelos MHF, Gonçales ES. Radiographic study of mandibular third molars: Evaluation of the position and root anatomy in Brazilian population. Oral Maxillofac Surg 2018; 22:163-8.

5. Patil SR, Araki K, Yadav N, Ghani HA. Prevalence of hypercementosis in a Saudi Arabian population: A cone beam computed tomography study. J Oral Res 2018; 7:94-7.

6. Dargue A. 'Acquired' concrescence causing surgical complications - a report of two cases. Oral Surg 2017; 10:e92-7.

7. Suter VGA, Reichart PA, Bosshardt DD, Bornstein MM. Atypical hard tissue formation around multiple teeth. Oral Surg Oral Med Oral Pathol Oral Radiol Endod 2011; 111:138-45.

8. Leider AS, Garbarino VE. Generalized hypercementosis. Oral Surg Oral Med Oral Pathol 1987; 63:375-80.

9. Olaf E. Langland, and Robert P. Langlais. Principles of Dental Imaging (Principles of dental imaging (Langland). Second Edi. Lippincott Williams and Wilkins; 2002.

10. Fragiskos FD. Oral Surgery. Vol $7^{\text {th }}$ ed. NewYork: Springer; 2007. 